\title{
Determinación de la Morfología del Canal Incisivo Mandibular Mediante Tomografía Computarizada de Haz Cónico (CBCT) en Población de Valdivia, Chile
}

\author{
Determination of Mandibular Incisor Canal Morphology by Cone Beam \\ Computed Tomography $(\mathrm{CBCT})$ in a Population of Valdivia, Chile
}

\author{
Gerardo Méndez ${ }^{1,2}$; Natalia Uribe ${ }^{1}$; Ximena Fuentes ${ }^{1} \&$ Guillermo Araya $^{1}$
}

\begin{abstract}
MÉNDEZ, G.; URIBE, N.; FUENTES, X. \& ARAYA, G. Determinación de la morfología del canal incisivo mandibular mediante tomografía computarizada de haz cónico (CBCT) en población de Valdivia, Chile. Int. J. Morphol., 39(5):1447-1452, 2021.

RESUMEN: El canal incisivo mandibular (MIC) es un canal neural que contiene una de las ramas inferiores del nervio alveolar inferior, llamado nervio incisivo mandibular, que puede resultar dañado durante intervenciones quirúrgicas y causar complicaciones postoperatorias. Estudio descriptivo de corte transversal. Se identificó el MIC en la imagen transversal del canino en 83 hemiarcadas. Se registró edad, sexo, hemiarcada, longitudes desde reborde alveolar vestibular de canino a MIC, cortical lingual y vestibular de canino a MIC, base mandibular de canino a MIC y ubicación del MIC (tercio lingual, medio, vestibular). Medidas se registraron en milímetros. Se aplicó test T-student para muestras independientes para variables de longitud y Chi-cuadrado para ubicación espacial del MIC, en relación con grupo etario y sexo. Se evaluó el MIC en todas las muestras (100 \%). El MIC fue encontrado mayormente en el tercio medio mandibular ( $\mathrm{p}<0,05$ ). La media desde el MIC a la cortical lingual es de 5,25 mm \pm 1,42 $\mathrm{mm}$ (derecho) y 5,24 $\mathrm{mm} \pm 1,18 \mathrm{~mm}$ (izquierdo). La media desde el MIC a la cortical vestibular fue de 4,42 $\mathrm{mm} \pm 1,29 \mathrm{~mm}$ (derecho) y 4,53 $\mathrm{mm} \pm 1,24 \mathrm{~mm}$ (izquierdo). La media entre centro del canal y reborde alveolar vestibular fue $18,89 \mathrm{~mm} \pm 2,68 \mathrm{~mm}$ (derecho) y 18,20 $\mathrm{mm} \pm 3,06 \mathrm{~mm}$ (izquierdo), media desde centro del MIC al margen basal fue de 9,77 $\mathrm{mm} \pm$ 1,93 (derecho) y 10,12 mm \pm 1,92 mm (izquierdo). Se encontró mayor distribución del MIC en el tercio medio mandibular. Se identificó el MIC en el $100 \%$ de las muestras a través de CBCT por lo que su uso como examen complementario debe ser considerado al planificar cirugías en el sector anterior mandibular.
\end{abstract}

PALABRAS CLAVE: Nervio mandibular; Implante dental; Tomografía computarizada Cone Beam; Anatomía; Canal incisivo mandibular.

\section{INTRODUCCIÓN}

Las cirugías en la zona anterior mandibular incluyen las cirugías de colocación de implantes, los injertos óseos y la genioplastia. Las cuales se consideran procedimientos quirúrgicos seguros y con riesgos estimados para los pacientes (Yang et al., 2017). Sin embargo, complicaciones postoperatorias como el dolor, parestesia, sangrado y trastornos sensoriales en la zona de inserción del implante dental en la región mandibular anterior se encuentran relacionados con la integridad del canal incisivo mandibular (Kong et al., 2016).

El canal incisivo mandibular (MIC) se describe como una prolongación del canal mandibular, anterior al foramen mental que contiene en su interior un haz neurovascular (Pires et al., 2012). El MIC contiene al nervio incisivo mandibular, el cual proporciona inervación a los dientes antero inferiores y a la mucosa circundante. El diámetro del canal mandibular disminuye de lateral a medial (Pietrokovski et al., 2007), al igual que su corticalización ósea (Kong et al.), produciendo que el MIC sea omitido.

En Chile se ha estudiado la presencia y morfología del MIC en radiografía panorámica, presentando limitaciones con respecto a la tomografía computarizada de haz cónico (CBCT) pudiendo subestimar los resultados respecto a su identificación y parámetros morfométricos (Fuentes et al., 2017). La presencia del canal incisivo mandibular ha sido observado en un $100 \%$ en disecciones cadavéricas (Xu et al., 2015) siendo, actualmente, la CBCT la mejor manera de evaluar la región maxilar-mandibular (Gomes et al., 2018), ya que permite la reconstrucción tridimensional de

\footnotetext{
${ }^{1}$ Escuela de Odontología, Facultad de Medicina, Universidad Austral de Chile.

${ }^{2}$ Instituto Anatomía, Histología y Patología. Facultad de Medicina, Universidad Austral de Chile.
} 
imágenes de alta resolución de toda la mandíbula, informándose excelentes imágenes de la mandíbula y el canal mandibular, junto a una alta precisión de las mediciones lineales, una baja dosis de radiación y un menor costo en comparación con la tomografía computarizada tradicional (Kong et al.). Ferreira Barbosa et al. (2019) en su revisión sistemática describe la identificación del canal incisivo mandibular en los estudios que va de un $71 \%$ al $100 \%$ de las muestras analizadas mediante CBCT, identificándose el MIC en el $89,6 \%$ de los estudios analizados.

La posición del canal mandibular (CM) y, por ende, el MIC como prolongación de éste pueden no ser constantes a través de la edad, cuya posición puede verse afectada por la remodelación ósea ocurrida con el envejecimiento (AlShayyab et al., 2019). Cambios en la densidad ósea también pueden dificultar la visibilidad de las estructuras (Jalili et al., 2012), lo que hace necesario un conocimiento preciso de la anatomía topográfica de la mandíbula para realizar adecuadamente los procedimientos en la región mandibular anterior (Gomes et al.). Es importante identificar a través de CBCT el canal incisivo mandibular o tener una referencia topográfica en casos donde el MIC no sea identificable a través de CBCT, con el fin de obtener un zona segura donde no haya riesgo de producir daño al haz neurovascular mandibular al momento de programar y realizar cirugías en la zona mandibular anterior.

El objetivo de este estudio fue determinar la morfología del canal incisivo mandibular y su ubicación mediante tomografía computarizada haz cónico (CBCT) en población chilena.

\section{MATERIAL Y MÉTODO}

Diseño de estudio y población estudiada. Se realizó un estudio descriptivo de corte transversal, para determinar la presencia y ubicación espacial del canal incisivo en el hueso mandibular. El cálculo de tamaño muestral se realizó a partir de la población de Valdivia (Chile) que corresponde según el censo 2017 a 166.080 habitantes, con software libre WinEpi 2.0 utilizando los siguientes datos: población conocida, prevalencia esperada de 89,6\% según lo descrito por Ferreira Barbosa et al. y con un error absoluto definido en un $10 \%$; el tamaño muestral calculado fue de 58 pacientes.

Imágenes CBCT. Se obtuvieron las imágenes de CBCT mandibulares de la Clínica Ramis Medicina, Odontología y Estética en Valdivia, Región de los Ríos (Chile) una clínica especializada en radiología maxilofacial en Valdivia (Chi- le) tomadas durante el periodo de enero 2018 y agosto 2020. Como criterio de inclusión para los pacientes seleccionados en este estudio se utilizó la ausencia de patología ósea o trauma mandibular, sin presencia de dientes supernumerarios o retenidos y mayores de 18 años con dentadura total o parcial que tuvieran el canino inferior presente. El protocolo para recoger la información fue aprobado por el Comité de Ética Científico del Servicio de Salud de Valdivia con orden $\mathrm{N}^{\circ} 158$ con fecha 15 de Junio del 2020.

Análisis CBCT. Para la visualización de imágenes se utilizó el software GALILEOS Viewer 1.9.2. (Denstplay Sirona). Los archivos DICOM fueron anonimizados previo utilización de estos, los únicos datos que se obtuvieron de los pacientes correspondieron a sexo y edad al momento de la recolección de datos. Se realizó calibración interexaminador con el $10 \%$ del tamaño muestral, un radiólogo bucomaxilofacial realizó dicho proceso con las investigadoras y se obtuvo un kappa de 0,79, luego de calibración se procedió a realizar las mediciones correspondientes. Se identificó el MIC en la imagen transversal a nivel de canino en 83 hemiarcadas. Se registró edad (años), sexo (masculino/femenino), hemiarcada (derecho/ izquierdo), longitud desde reborde alveolar vestibular de canino a MIC, longitud horizontal desde cortical lingual y vestibular del canino a MIC, longitud vertical desde base mandibular de canino a MIC y ubicación del canal incisivo (lingual, medio, vestibular) (Fig. 1), esto fue registrado en un gestor de formularios Google Drive TM, el cual sirvió de base de datos. Las medidas se registraron en milímetros.

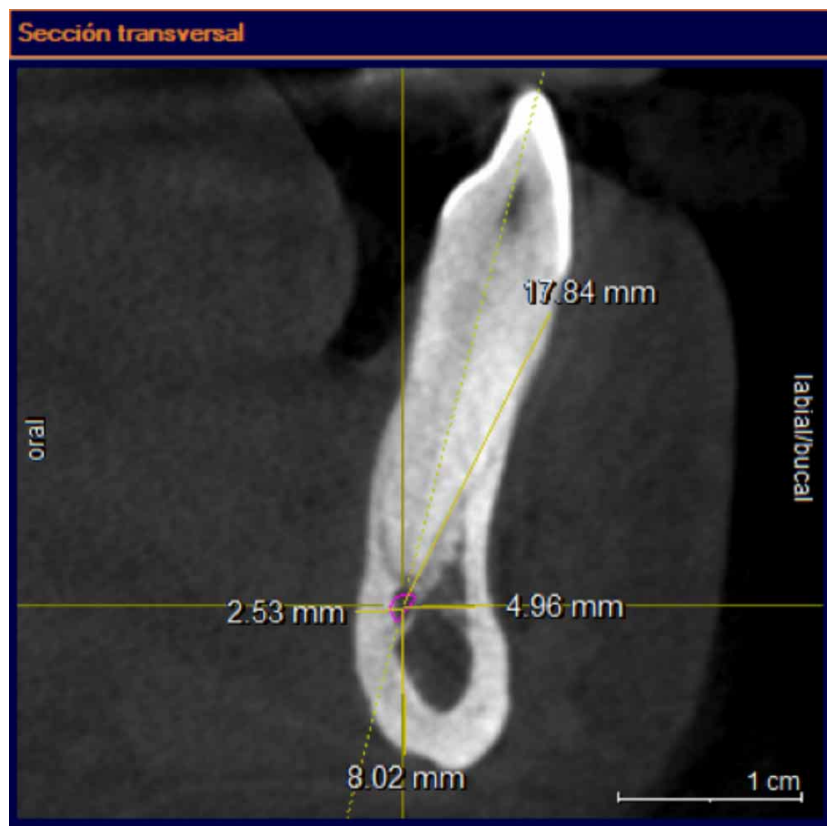

Fig. 1. Vista transversal a nivel de canino inferior derecho. Medidas en milímetros de las distancias desde centro del canal incisivo mandibular a margen vestibular, lingual, inferior mandibular y reborde alveolar vestibular de canino. 
Análisis estadístico. Se realizó el análisis estadístico mediante el software de InfoStat versión libre acceso (InfoStat/ L) año 2019 y RStudio (versión 1.3.1073) en lenguaje $r$ (valor $\mathrm{p}<0,05$ ). Para variables con distribución normal se aplicó test T-student para muestras independientes, para las variables no paramétricas se realizó chi-cuadrado para analizar diferencia entre grupo etario, sexo y ubicación espacial del MIC.

\section{RESULTADOS}

Se estudiaron 83 imágenes CBCT de hemiarcadas, 25 pertenecientes a hombres y 58 a mujeres, se evaluó el MIC en todas las muestras (100\%). En la Tabla I se muestra la distribución por edad y ubicación espacial del MIC por sexo. Se distribuyeron los pacientes por rango de edad y sexo. La ubicación espacial del canal incisivo mandibular se dividió en tres tercios: lingual, medio y vestibular según sexo. En la Figura 2 se representa en un gráfico la ubicación distribuida en tercios respecto al total de datos, donde se muestra una distribución mayor del MIC en el tercio medio mandibular teniendo significancia estadística ( $p$ $<0,05)$.

El gráfico de la Figura 3 presenta la dispersión de datos respecto a la edad con las longitudes medidas en relación a el MIC, no se encontró diferencia estadística significativa entre variación de longitud y edad ( $p>0,05)$, siguiendo una distribución lineal. La Tabla II describe la distancia media y desviación estándar entre el centro del canal incisivo mandibular a reborde alveolar vestibular, cortical vestibular, lingual y basal de canino obtenidas por sexo y lado mandibular junto a los valores mínimos y máximos. La distancia media horizontal desde el centro del MIC a la cortical lingual y vestibular fue de $5,25 \mathrm{~mm} \pm 1,42 \mathrm{~mm}$ y 4,42 $\pm 1,29 \mathrm{~mm}$ respectivamente en el lado derecho y de $5,24 \mathrm{~mm} \pm 1,18 \mathrm{~mm}$ y $4,53 \mathrm{~mm} \pm 1,24 \mathrm{~mm}$ en el lado izquierdo, la distancia mínima registrada entre la cortical vestibular y el MIC fue de 2,14 mm en el lado derecho y $2,68 \mathrm{~mm}$ en el lado izquierdo, mientras que la distancia mínima entre la cortical lingual y el MIC fue de $1,97 \mathrm{~mm}$ en el lado derecho y $2,04 \mathrm{~mm}$ en el lado izquierdo. La distancia media entre el centro del canal y el reborde alveolar vestibular fue de $18,89 \mathrm{~mm} \pm 2,68 \mathrm{~mm}$ en el lado derecho y $18,20 \mathrm{~mm} \pm 3,06 \mathrm{~mm}$ en el lado izquierdo, teniendo como valores mínimos $13,03 \mathrm{~mm}$ y $4,97 \mathrm{~mm}$ respectivamente, mientras que la distancia vertical desde el centro del MIC al margen basal fue de $9,77 \mathrm{~mm} \pm 1,93 \mathrm{~mm}$ en el lado derecho y $10,12 \mathrm{~mm} \pm 1,92 \mathrm{~mm}$ en el lado izquierdo.

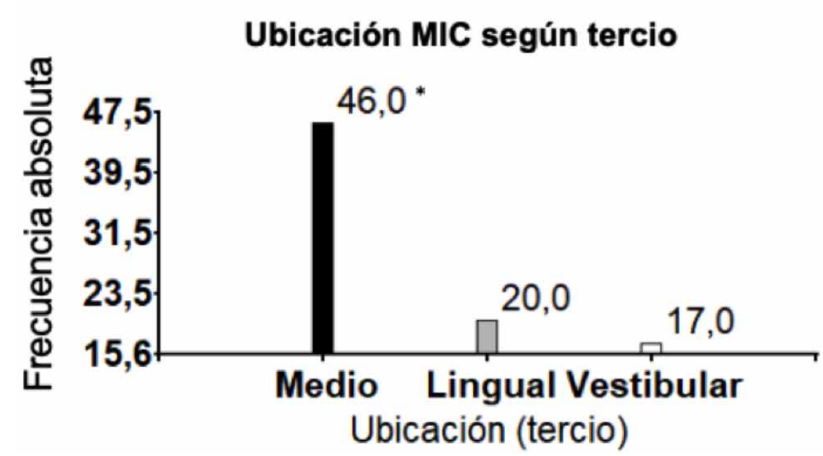

Fig. 2. Distribución espacial del canal incisivo mandibular (MIC) según su ubicación en tercio de sección transversal del hueso mandibular a nivel de canino respecto a frecuencia absoluta del total de edades. * Diferencia estadísticamente significativa según prueba chi-cuadrado $(\mathrm{p}<0.05)$

Tabla I. Valores de edad respecto al sexo y ubicación espacial del canal incisivo mandibular a nivel de canino.

\begin{tabular}{|c|c|c|c|c|}
\hline & Sexo & & & $x^{2}$ \\
\hline & $\begin{array}{c}\text { Masculino } \\
(\%)\end{array}$ & $\begin{array}{c}\text { Femenino } \\
(\%)\end{array}$ & $\begin{array}{c}\text { Total } \\
(\%)\end{array}$ & \\
\hline \multicolumn{5}{|l|}{ Edad } \\
\hline $18-38$ & $4(16,00)$ & $29(50,00)$ & $33(39,76)$ & \\
\hline $39-59$ & $17(68,00)$ & $21(36,21)$ & $38(45,78)$ & \\
\hline $60-80$ & $4(16,00)$ & $8(13,79)$ & $12(14,46)$ & \\
\hline $\mathrm{n}$ & 25 & 58 & 83 & \\
\hline \multicolumn{5}{|l|}{ Ubicación } \\
\hline Tercio lingual & $10(40,00)$ & $10(17,24)$ & $20(24,10)$ & \\
\hline Tercio medio & $11(44,00)$ & $35(60,35)$ & $46^{*}(55,42)$ & $\begin{array}{c}<0.00 \\
1\end{array}$ \\
\hline $\begin{array}{l}\text { Tercio } \\
\text { vestibular }\end{array}$ & $4(16,00)$ & $13(22,41)$ & $17(20,48)$ & \\
\hline
\end{tabular}

$\mathrm{x}^{2} ;$ prueba chi-cuadrado $*$ diferencia estadística $(\mathrm{p}<0.05)$ 
Tabla II. Distancia media y desviación estándar (DE) entre el centro del canal incisivo mandibular a reborde alveolar vestibular, cortical vestibular, lingual y basal de canino obtenidas por sexo y lado mandibular.

\begin{tabular}{|c|c|c|c|c|c|c|c|c|c|}
\hline \multirow[b]{2}{*}{$\begin{array}{l}\text { Lado } \\
\text { Derecho } \\
(\mathrm{mm})\end{array}$} & \multicolumn{2}{|c|}{ Sexo } & \multirow[t]{2}{*}{$\mathrm{pt}$} & \multicolumn{2}{|l|}{ TOTAL } & \multicolumn{2}{|c|}{ Sexo } & \multirow[t]{2}{*}{$\mathrm{pt}$} & \multirow{2}{*}{$\begin{array}{c}\text { TOTAL } \\
\text { Media (DE) } \\
{[\min -\max ]}\end{array}$} \\
\hline & $\begin{array}{l}\text { Masculino } \\
\text { Media (DE) }\end{array}$ & $\begin{array}{c}\text { Femenino } \\
\text { Media (DE) }\end{array}$ & & $\begin{array}{l}\text { Media (DE) } \\
{[\min -\max ]}\end{array}$ & $\begin{array}{c}\text { Lado } \\
\text { Izquier do } \\
(\mathrm{mm})\end{array}$ & $\begin{array}{l}\text { Masculino } \\
\text { Media (DE) }\end{array}$ & $\begin{array}{c}\text { Femenino } \\
\text { Media (DE) }\end{array}$ & & \\
\hline $\mathrm{n}$ & 13 & 31 & & 44 & & 12 & 27 & & 39 \\
\hline Reborde & $19,48(2,81)$ & $18,64(2,63)$ & 0,349 & $\begin{array}{c}18,89(2,68) \\
{[13,03-25,56]}\end{array}$ & & $18,88(2,44)$ & $17,90(3,29)$ & 0,362 & $\begin{array}{c}18,20(3,06) \\
{[4,97-23,13]}\end{array}$ \\
\hline Vestibular & $4,91(1,48)$ & $4,22(1,17)$ & 0,105 & $\begin{array}{c}4,42(1,29) \\
{[2,14-7,22]}\end{array}$ & & $5,15(1,49)$ & $4,25(1,03)$ & $0,034 *$ & $\begin{array}{c}4,53(1,24) \\
{[2,68-7,52]}\end{array}$ \\
\hline Lingual & $5,27(1,63)$ & $5,24(1,35)$ & 0,965 & $\begin{array}{c}5,25(1,42) \\
{[1,97-9,16]}\end{array}$ & & $5,01(1,33)$ & $5,34(1,12)$ & 0,782 & $\begin{array}{c}5,24(1,18) \\
{[2,04-7,91]}\end{array}$ \\
\hline Basal & $10,08(2,35)$ & $9,64(1,75)$ & 0,495 & $\begin{array}{c}9,77(1,93) \\
{[6,54-15,85]}\end{array}$ & & $10,27(2,16)$ & $10,05(1,85)$ & 0,749 & $\begin{array}{c}10,12(1,92) \\
{[6,31-14,08]}\end{array}$ \\
\hline
\end{tabular}

Pt, t test; *, diferencia estadística $(\mathrm{p}<0.05)$.

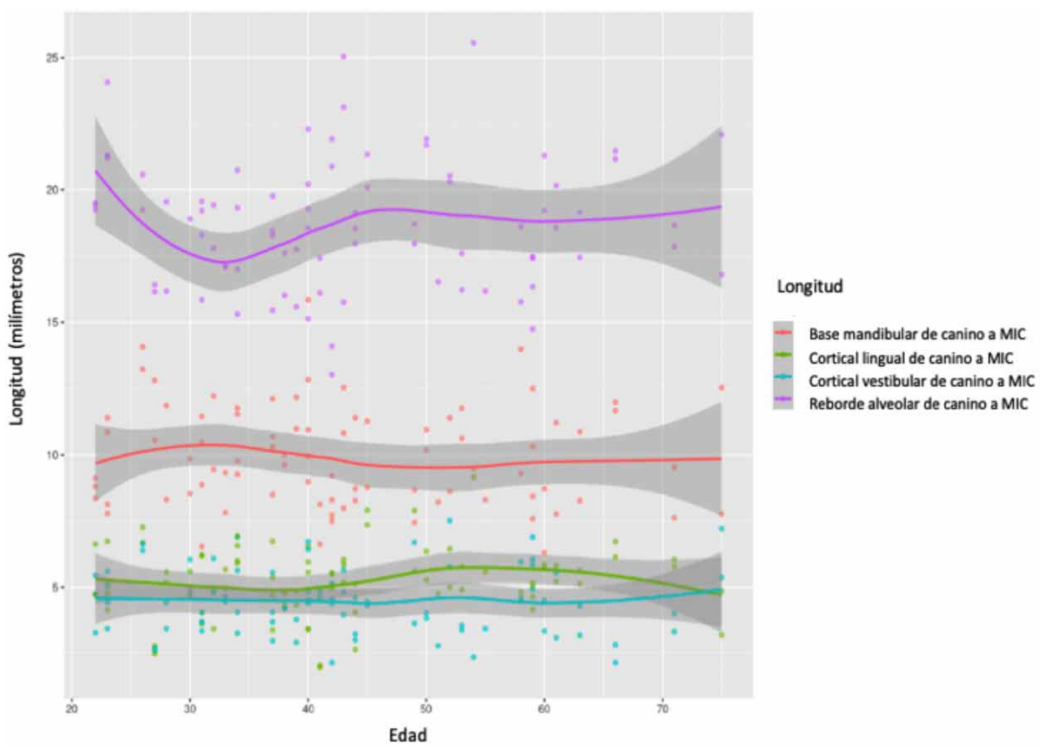

Fig. 3. Dispersión en relación a la edad con las longitudes medidas en relación a el canal incisivo mandibular (MIC).

que se quejó de dolor y entumecimiento del tejido circundante durante más de 1 año después de que se extrajo un injerto óseo de la barbilla derecha, en cuyas imágenes de CBCT, la lesión en el MIC derecho fue evidente en el sitio de recolección.

Se ha estudiado el uso de CBCT para la evaluación del canal incisivo mandibular, presentando porcentajes que van de un 71 $\%$ al $100 \%$ de identificación del MIC en las muestras de CBCT analizadas con una prevalencia promedio de $89,6 \% \pm 15,08 \%$ (Ferreira Barbosa et al.). En nuestro estudio se identificó el MIC en el $100 \%$ de las muestras, Kong et al. también identificó en $100 \%$ de las imágenes CBCT el MIC y con buena definición en el 63,6\%., Pires et al. observó la presencia del MIC en un $83 \%$ de los CBCT evaluados, siendo la radiografía panorámica la que presenta menores

\section{DISCUSIÓN}

Estudios describen trastornos sensoriales y fracasos clínicos después de cirugías relacionadas con la zona anterior mandibular (Kong et al.). Gomes et al. en su estudio evaluó cincuenta y cinco pacientes con sospecha de relación entre el canal incisivo mandibular y los implantes instalados. Los exámenes de tomografía computarizada revelaron que 10 pacientes con dolor neuropático mostraron perforación del canal incisivo en al menos un implante por persona. Kong et al. en su estudio muestra las imágenes de CBCT correspondiente a una paciente de 56 años que se quejó de dolor durante 7 meses después de que se insertaron los implantes en zona incisiva mandibular; En la imagen, se descubrió que el implante izquierdo estaba en relación con el MIC evidenciando pérdida cortical de éste. También, muestra las imágenes de un paciente masculino de 34 años valores de identificación del MIC (51,2 \%) (Sahman et al., 2014), mientras que en estudios de disección de cadáveres ha sido observado en un $100 \%$ (Xu et al.).

Se ha descrito la cercanía del MIC con la cortical vestibular y lingual, donde Lim et al. (2019) describe que a nivel inicial el canal incisivo mandibular se encuentra más cercano a la cortical vestibular y a nivel final y medio más cercano a la cortical lingual, sin embargo, a nivel general el curso del MIC se inclinó más hacia el margen vestibular de la mandíbula. Pires et al., en cambio, describe que el MIC se encontraría más cerca de la cortical vestibular independientemente de su origen o ubicación terminal, Kong et al. también describe una cercanía del canal con el margen vestibular y margen inferior mandibular al igual que $\mathrm{Vu}$ et al. (2015) en su estudio de disección de cadáveres. En nuestro estudio se dividió el hueso mandibular en tres tercios en sentido transversal, encontrando una mayor distribución del MIC en el tercio medio mandibular ( $\mathrm{p}<0,05$ ) (Fig. 3). 
A partir de los resultados se concluye que no hubo diferencia estadística significativa en la diferencia de longitudes entre sexo y edad ( $\mathrm{p}>0,05)$, como tampoco entre sexo y ubicación espacial ( $\mathrm{p}>0,05)$. Kabak et al. (2016) afirma también que no habría diferencia estadística significativa entre el diámetro del MIC y los lados derecho e izquierdo o entre pacientes masculinos y femeninos.

Las longitudes promedio en relación al MIC son similares a las encontradas en otros estudios con algunas diferencias, Kong et al. en población china a nivel de canino describieron una distancia media desde MIC a cortical lingual y vestibular de $5,11 \mathrm{~mm} \pm 1,43 \mathrm{~mm}$ y $4,65 \mathrm{~mm} \pm 1,28 \mathrm{~mm}$ respectivamente y desde margen inferior a MIC de 8,21 mm $\pm 2,15 \mathrm{~mm}$, mientras que la distancia promedio a margen inferior registrada en nuestro estudio fue de 9,77 $\mathrm{mm} \pm 1,93$ mm lado derecho y $10,12 \mathrm{~mm} \pm 1,92 \mathrm{~mm}$ lado izquierdo, al igual que la distancia promedio de cresta alveolar a MIC fue de $20,44 \mathrm{~mm} \pm 3,26 \mathrm{~mm}$ mientras que en nuestro estudio fue de $18,89 \mathrm{~mm} \pm 2,68 \mathrm{~mm}$ el lado derecho y 18,20 $\mathrm{mm} \pm 3,06 \mathrm{~mm}$ el lado izquierdo.

Yang et al., en su estudio en población china, describieron una distancia promedio desde la cortical lingual de 4,98 $\mathrm{mm} \pm 1,30 \mathrm{~mm}$ y vestibular de 4,91 $\mathrm{mm} \pm 1,45 \mathrm{~mm}$ a nivel de canino similares a las registradas en nuestro estudio con valores mínimos y máximos encontrados en vestibular de $1,5 \mathrm{~mm}$ a 9,3 $\mathrm{mm}$ y lingual de $1,3 \mathrm{~mm}$ a 10,3 $\mathrm{mm}$ mientras que nuestro estudio registró valores en vestibular de 2,14 $\mathrm{mm}$ a 7,52 $\mathrm{mm}$ y lingual 1,97 $\mathrm{mm}$ a 9,16 $\mathrm{mm}$, a nivel de margen inferior describió una distancia promedio de $7,95 \mathrm{~mm} \pm 1,84 \mathrm{~mm}$ y valores entre $2,43 \mathrm{~mm}$ a $17,01 \mathrm{~mm}$ variando con el promedio registrado en nuestro estudio $(9,77 \mathrm{~mm} \pm 1,93 \mathrm{~mm}$ lado derecho y $10,12 \mathrm{~mm} \pm$ $1,92 \mathrm{~mm}$ lado izquierdo) y presentando también una menor distancia mínima y mayor distancia máxima registrada $(6,31$ $\mathrm{mm}$ a $15,85 \mathrm{~mm}$ en nuestro estudio), a nivel de cresta alveolar registró distancias de 19,53 $\mathrm{mm} \pm 2,63 \mathrm{~mm}$ con valores entre $11,61 \mathrm{~mm}$ a $26,37 \mathrm{~mm}$, mientras que en nuestro estudio la menor distancia registrada a reborde alveolar a MIC fue de 4,97 $\mathrm{mm}$.

Kabak et al. encontró longitudes promedio a nivel de canino de 3,9 mm entre MIC y cortical vestibular con valores mínimos y máximos de 1,4 mm a 8,0 mm, 4,4 mm a cortical lingual con valores entre $1,3 \mathrm{~mm}$ a $10 \mathrm{~mm}$, presentando valores mínimos menores a los registrados $(2,14 \mathrm{~mm}$ vestibular y $1,97 \mathrm{~mm}$ lingual) y distancia de MIC a margen inferior de 8,5 $\mathrm{mm}$, con valores entre $5,3 \mathrm{~mm}$ a $14,9 \mathrm{~mm}$, no mostrando una diferencia significativa en valores promedio con nuestro estudio. En un estudio en 19 disecciones de cadáveres ( $\mathrm{Vu}$ et al.) en población estadounidense se encontraron longitudes en el lado derecho mandibular entre MIC y cortical vestibular de 4,18 $\mathrm{mm} \pm 1,78 \mathrm{~mm}$, de 4,77 $\mathrm{mm} \pm$ $1,61 \mathrm{~mm}$ a cortical lingual, $10,18 \mathrm{~mm} \pm 2,18 \mathrm{~mm}$ a margen inferior mandibular y entre MIC y unión cemento esmalte de $22,37 \mathrm{~mm} \pm 3,78 \mathrm{~mm}$, mientras que en lado izquierdo los valores fueron de $3,55 \mathrm{~mm} \pm 1,56 \mathrm{~mm}$ a nivel vestibular, $4,81 \mathrm{~mm} \pm 2,02 \mathrm{~mm}$ a nivel lingual, $10,59 \mathrm{~mm} \pm 1,74 \mathrm{~mm}$ margen inferior y $20,38 \mathrm{~mm} \pm 5,82 \mathrm{~mm}$ a unión cemento esmalte, mostrando valores similares respecto a los registrados en nuestro estudio.

El canal incisivo mandibular ha sido estudiado en Chile en radiografías panorámicas siendo identificado en un 35,9\% (Fuentes et al., 2015) y $53 \%$ (Fuentes et al., 2017) de las radiografías estudiadas, a diferencia de nuestro estudio en CBCT donde se identificó el MIC en un $100 \%$ de las muestras. En el estudio de Fuentes et al. (2017) se encontraron valores subestimados en comparación con los resultados obtenidos en estudios con $\mathrm{CBCT}$, siendo los análisis con CBCT más eficientes tanto en la identificación como en la evaluación del MIC. En comparación con nuestro estudio Fuentes et al. (2017) registró una distancia media del MIC al margen inferior mandibular de $9,1 \mathrm{~mm} \pm 1,8 \mathrm{~mm}$, con valores que variaron de $4,1 \mathrm{~mm}$ a $16,7 \mathrm{~mm}$, mientras que en nuestro estudio se registró una variación de $6,31 \mathrm{~mm}$ a $15,85 \mathrm{~mm}$ y una media de $9,77 \mathrm{~mm} \pm 1,93 \mathrm{~mm}$ en lado derecho y $10,12 \mathrm{~mm} \pm 1,92 \mathrm{~mm}$ en el lado izquierdo. $\mathrm{Si}$ bien los promedios registrados pueden ser similares entre los estudios, es importante tener en consideración la variación significativa que presentan las distancias mínimas y máximas registradas para su aplicación clínica, que puede ser importante debido al riesgo de daño neurovascular al momento de la realización de cirugías en el sector anterior mandibular. En nuestro estudio la distancia mínima registrada desde el reborde alveolar vestibular al centro del MIC fue de 4,97 mm, a nivel vestibular y lingual la distancia al MIC mínima registrada fue de 2,14 $\mathrm{mm}$ y 2,68 mm respectivamente mientras que de MIC a margen inferior la distancia mínima fue de $6,31 \mathrm{~mm}$, lo que al momento de realizar una cirugía de implantes puede ser relevante, al igual que en cirugías de injerto y genioplastia que involucran la zona anterior mandibular.

Es importante tener en consideración la relación con las estructuras neurovasculares al momento de realizar cirugías en el sector anterior mandibular y al evaluar el espacio disponible para la colocación de implantes con el fin de minimizar el riesgo de daño al haz neurovascular y las complicaciones postoperatorias que esto conlleva.

Como limitaciones de este estudio podemos mencionar que no se incluyeron pacientes desdentados en la muestra. Para estudios futuros sería ideal usar otros puntos de referencia además del canino e incluir pacientes desdentados. 
El uso de CBCT permite realizar una evaluación dimensional y topográfica del MIC, por lo que su uso como examen complementario debe ser considerado al planificar cirugías en el sector anterior mandibular. Se identificó el MIC en un $100 \%$ de las muestras con el uso de CBCT y se encontró una distribución mayor del MIC en el tercio medio mandibular en sentido transversal, no se encontró relación entre ubicación y edad en pacientes dentados.

AGRADECIMIENTOS. Esta investigación está basada en una tesis realizada por Natalia Uribe Loebel y Ximena Fuentes Gajardo como requerimiento para la obtención del grado académico de Licenciado en Odontología de la Universidad Austral de Chile, Noviembre, 2020.

MÉNDEZ, G.; URIBE, N.; FUENTES, X. \& ARAYA, G. Determination of mandibular incisor canal morphology by cone beam computed tomography (CBCT) in a population of Valdivia, Chile. Int. J. Morphol., 39(5):1447-1452, 2021.

SUMMARY: The objective of the study was to determine the morphology of the mandibular incisive canal (MIC) and its location using cone beam computed tomography (CBCT) in the population of Valdivia, Chile. Descriptive cross-sectional study. MIC was identified in the canine cross image in 83 quadrants. Age, gender, quadrants, length from buccal alveolar ridge of canine to MIC, lingual and buccal cortical of canine to MIC, mandibular base of canine to MIC, and location of MIC (lingual, middle and buccal third) were recorded. Measurements were recorded in millimeters. Independent sample Student-T test was performed to determine length variables and Chi-square test was performed to determine spatial location of $\mathrm{MIC}$, in relation to age group and gender. MIC was evaluated in all samples $(100 \%)$. MIC was found mainly in the mandibular third quadrant $(\mathrm{p}<0.05)$. The mean from the MIC to the lingual cortex is $5.25 \mathrm{~mm} \pm 1.42 \mathrm{~mm}$ (right) and $5.24 \mathrm{~mm} \pm 1.18 \mathrm{~mm}$ (left). The mean from the MIC to the buccal cortex was $4.42 \pm 1.29 \mathrm{~mm}$ (right) and $4.53 \mathrm{~mm} \pm 1.24 \mathrm{~mm}$ (left). The mean between the center of the canal and the buccal alveolar ridge was $18.89 \mathrm{~mm} \pm 2.68 \mathrm{~mm}$ (right) and $18.20 \mathrm{~mm} \pm 3.06 \mathrm{~mm}$ (left), the mean from the center of the MIC to the basal edge was $9.77 \mathrm{~mm} \pm 1.93$ (right) and $10.12 \mathrm{~mm} \pm 1.92 \mathrm{~mm}$ (left). A greater distribution of MIC was found in the mandibular third quadrant. MIC was identified in $100 \%$ of the samples through CBCT, therefore, its use as a complementary examination should be considered when planning surgeries in the anterior mandibular area.

KEY WORDS: Mandibular nerve; Dental implant; Cone Beam computed tomography; Anatomy; Mandibular incisive canal.

\section{REFERENCIAS BIBLIOGRÁFICAS}

Al-Shayyab, M. H.; Qabba'ah, K.; Alsoleihat, F. \& Baqain, Z. H. Age and gender variations in the cone-beam computed tomographic location of mandibular canal: Implications for mandibular sagittal split osteotomy. Med. Oral Patol. Oral. Cir Bucal, 24(4):e545-54, 2019.
Ferreira Barbosa, D. A.; Barros, I. D. \& Teixeira, R. C. Imaging aspects of the mandibular incisive canal: A PROSPERO-registered systematic review and meta-analysis of cone beam computed tomography studies. Int. J. Oral Maxillofac. Implants, 34(2):423-33, 2019.

Fuentes, R.; Arias, A.; Bucchi, C.; Saravia, D. \& Dias, F. Prevalence and morphometric characteristics of the mandibular incisive canal through panoramic radiographs in a Chilean population. Int. J. Morphol., 35(3):9317, 2017.

Fuentes, R.; Bucchi, C.; Navarro, P.; Beltran, V. \& Cantin, M. Characterization of anatomical structures using panoramic X-rays: Part II: Mandibular incisive canal. J. Anat. Soc. India., 64(2):128-32, 2015.

Gomes, L. T.; de Almeida Barros Mourão, C. F.; Braga, C. L.; Duarte de Almeida, L. F.; Coutinho de Mello-Machado, R. \& Calasans-Maia, M. D. Anatomic evaluation of the incisive canal with cone beam computed tomography and its relevance to surgical procedures in the mental region: a retrospective study in a Brazilian population. Oral Maxillofac. Surg., 22(4):379-84, 2018.

Jalili, M. R.; Esmaeelinejad, M.; Bayat, M. \& Aghdasi, M. M. Appearance of anatomical structures of mandible on panoramic radiographs in Iranian population. Acta Odontol. Scand., 70(5):384-9, 2012.

Kabak, S. L.; Zhuravleva, N. V.; Melnichenko, Y. M. \& Savrasova, N. A. Study of the mandibular incisive canal anatomy using cone beam computed tomography. Surg. Radiol. Anat., 39(6):647-55, 2016.

Kong, N.; Hui, M.; Miao, F.; Yuan, H.; Du, Y. \& Chen, N. Mandibular incisive canal in Han Chinese using cone beam computed tomography. Int. J. Oral Maxillofac. Surg., 45(9):1142-6, 2016.

Lim, J.; Kang, W. J.; Ajit Bapat, R.; Kanneppady, S. K. \& Pandurangappa, R. Evaluation of mandibular incisive canal using cone beam computed tomography in Malaysians. J. Maxillofac. Oral Surg., 18(4):596-603, 2019.

Pietrokovski, J.; Starinsky, R.; Arensburg, B. \& Kaffe, I. Morphologic characteristics of bony edentulous jaws. J. Prosthodont., 16(2):141-7, 2007.

Pires, C. A.; Bissada, N. F.; Becker, J. J.; Kanawati, A. \& Landers, M. A. Mandibular incisive canal: cone beam computed tomography. Clin. Implant Dent. Relat. Res., 14(1):67-73, 2012.

Sahman, H.; Sekerci, A. E.; Sisman, Y. \& Payveren, M. Assessment of the visibility and characteristics of the mandibular incisive canal: cone beam computed tomography versus panoramic radiography. Int. J. Oral Maxillofac. Implants, 29(1):71-8, 2014.

Vu, D. D.; Brockhoff 2nd, H. C.; Yates, D. M.; Finn, R. \& Phillips, C. Course of the mandibular incisive canal and its impact on harvesting symphysis bone grafts. J. Oral Maxillofac. Surg., 73(2):258.e1-258.e12, 2015.

Xu, Y.; Suo, N.; Tian, X.; Li, F.; Zhong, G.; Liu, X.; Bao, Y.; Song, T. \& Tian, $\mathrm{H}$. Anatomic study on mental canal and incisive nerve canal in interforaminal region in Chinese population. Surg. Radiol. Anat., 37(6):585-9, 2015.

Yang, X. W.; Zhang, F. F.; Li, Y. H.; Wei, B. \& Gong, Y. Characteristics of intrabony nerve canals in mandibular interforaminal region by using conebeam computed tomography and a recommendation of safe zone for implant and bone harvesting. Clin. Implant Dent. Relat. Res., 19(3):530-8, 2017.

\section{Dirección para correspondencia: \\ Gerardo Méndez \\ Isla Teja $\mathrm{S} / \mathrm{N}$ \\ Facultad de Medicina \\ Universidad Austral de Chile \\ Valdivia \\ CHILE}

E-mail: gerardo.mendez@uach.cl

Recibido : 12-05-2021

Aceptado : 30-06-2021 